\title{
The fabrication and modelling of non-silica microstructured optical fibres
}

Daniel W. Hewak, Yvonne D. West, N.G.R. Broderick,

Tanya M. Monro, and D.J. Richardson

Optoelectronics Research Centre, University of Southampton, Southampton, S017 JBJ, UK

We present for the first time a non-silica microstructured optical fibre designed to guide light at $5 \mu \mathrm{m}$ using gallium lanthanum suphide glass. We present the results of optical modelling of the structure as well as a discussion on possible applications.

\section{Introduction}

Silica is perhaps the ideal passive medium for optical communications, however its very inertness makes active applications, that is, direct manipulation of the light within the fibre, difficult. There are a whole range of lasing and amplifying transitions, across the telecommunications transmission windows, which cannot be achieved in a silica glass host. Moreover, by virtue of its inherent stability, electro-optic, acousto-optic and magneto-optic interactions, nonlinear and photorefractive effects are weak in silica glass. Hence to develop active all-optical devices such as routers and switches suitable for the next generation of all-optical communications systems novel non-silica glasses must be used[1].

Gallium lanthanum sulphide (GLS) glass offers a range of important properties which can be exploited for active device applications. GLS is transparent for wavelengths up to 5 microns, has a high refractive index (2.3 - 2.5), and can be doped with over $10 \%$ rare earth ions by weight [2]. However, GLS glass is difficult to form into a traditional optical fibre structure mainly due to the different physical properties of the core and cladding materials. This paper presents the marriage of our efforts in microstructured fibre and novel glass technology.

Microstructured or 'holey' fibres offer an almost unparalleled flexibility over the control of the basic fibre parameters due to the freedom in choosing the basic structure as well as the large index differences whith are possible in air-silica structures. Although it has long been believed that such fibres could be made using almost any glass until very recently no attempt has been made to fabricate such structures. In this paper we analyse the optical properties of a recent gallium lanthanum suphide (GLS) based microstructured fibre and then using this knowledge we discuss the future possibilities of such fibres.

\section{Fabrication}

The most significant difference between silica and non-silica ( compound glasses) are the radical differences in the thermal properties and hence the fabrication methods used to prepare the glass and draw a fibre. Although GLS glass has relatively high characteristic temperatures compared to other non-silica glasses, its melting temperature is on the order of $840^{\circ} \mathrm{C}$ compare to over $2200^{\circ} \mathrm{C}$ for silica. The coefficient of thermal expansion is not negligible as it is with silica, however relatively low for compound glasses. Fibre drawing temperature of GLS glass is only around $700^{\circ} \mathrm{C}$ and is very close to the crystallization temperature as shown in Fig. 1a. This places strict requirements on temperature control during the fibre drawing process.

The binary gallium lanthanum sulphide system has a maximum stability for a gallium to lanthanum ratio around 70:30[3] . Batches of powders are placed in a vitreous carbon crucible and melted in a tube furnace at $1150^{\circ} \mathrm{C}$ for typically $24 \mathrm{hrs}$ depending on the batch size. The resulting melts are then rapidly cooled to form the glass. After quenching the resulting glass ingots glasses are then annealed near the glass transition temperature and can then be formed into the desired rods and tubes from which an optical fibre preform is assembled. 
Figure 1b shows a cross-section of a GLS microstructured fibre designed to guide at $5 \mu \mathrm{m}$. This fibre was drawn from the preform in a single step, and this preform was constructed in a manner analogous to conventional air-silica microstructured preforms[4]. A solid rod was used to form the core of the fibre, this rod was surrounded by six capillaries, and this structure was placed inside a larger capillary both for structural support and to increase the preform dimensions. This preform was then drawn into fibre form. We note that it was obvious from the preform that any modes of the resulting fibre would be leaky due to both the limited number of capillaries used and their dimensions. However by drawing such a fibre we would learn many crucial details such as the degree of collapse and what the useful temperature range would be. In addition the optical properties of the fibre could tell us useful information about the loss and whether or not there was a significant degree of crystallisation on the walls of the capillaries (which is a major problem when drawing step index GLS fibres).

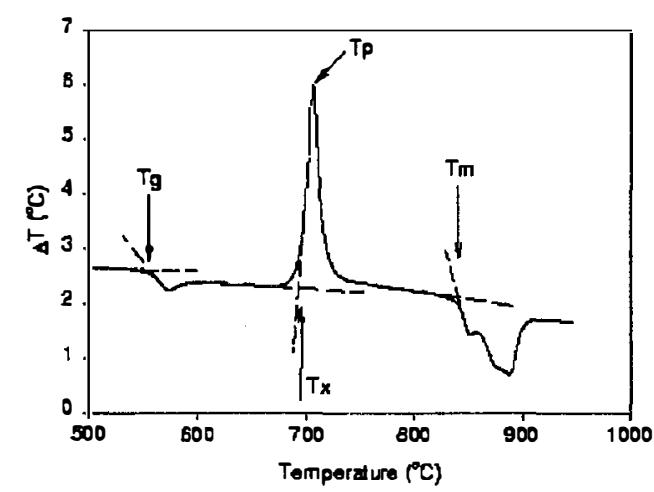

(a) Typical DTA curve

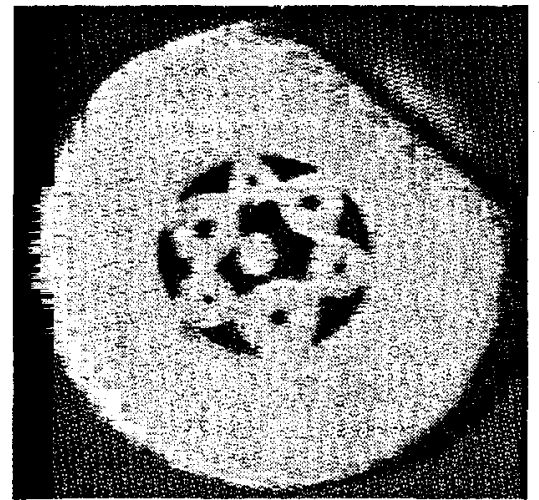

(b) GLS microstructured fibre

Fig. 1. (a) Thermal analysis of GLS glass showing the glass transition temperature $T_{g}$ the crystalisation temperature $T_{x}$, crystalisation peak $T_{p}$ and the melting temperature $T_{m}$. (b) A GLS (gallium lanthanum suphide) microstructured optical fibre. The core is supported by capillaries, which form the cladding region.

The fibre Fig. 1b has an outer diameter of approximately $100 \mu \mathrm{m}$, and a core diameter of $10 \mu \mathrm{m}$. The holes in the cladding capillaries range in diameter from 1.5 to $4.0 \mu \mathrm{m}$. In this case the inner capillaries did net fit tightly in the supporting capillary, which resulted in the core being loosely attached to the cladding. Figure 1b shows that it is possible to retain the transverse structure during fibre drawing and improved more highly structured preforms are now being prepared. The excellent structure retention we have obtained indicates that the viscosity/temperature relationship in GLS glass is well suited to microstructured fibre fabrication.

\section{Optical properties}

The unusual properties of microstructured fibres result from the fact that the structure in the cladding can be on the same physical scale as the wavelength of light. Compound glass microstructured fibres have potential applications which include: transmission at wavelengths from the visible to beyond $5 \mu \mathrm{m}$, large mode sizes, highly nonlinearity, novel dispersion characteristics, photonic band gap guidance, endless single-mode guidance, large evanescent fields, amongst others.

To model the optical properties of a microstructured fibre, it is necessary to describe the transverse refractive index profile accurately. This can be done using a full vector model in which the guided modes and the index profile are described independently [5, 6]. The GLS fibre shown in Fig. $1 \mathrm{~b}$ can be modeled directly using this approach. Figure 2a shows that this fibre supports a guided mode in the core at $4 \mu \mathrm{m}$. This mode is somewhat leaky due to the thickness of the bridge between the core and cladding regions. The core also supports two higher order leaky modes.

Consider an idealised GLS microstructured fibre with a hole-to-hole spacing of $5 \mu \mathrm{m}$ and holes of size $1 \mu \mathrm{m}$ 
arranged in a hexagonal configuration. Note by reference to Fig. $1 \mathrm{~b}$ that features of these dimensions can be achieved. The numerical model predicts that this fibre will be single-moded from at least $1.0 \mu \mathrm{m}$ to $5.0 \mu \mathrm{m}$. The predicted mode field diameter (MFD) and waveguide dispersion (GVD) for this fibre are given in Fig. 2b. Both the MFD and the GVD are relatively flat over this large wavelength range. The increase in the MFD at short wavelengths results from the fact that the guided mode then becomes extended in the regions between the holes. The waveguide GVD is small and anomalous over the entire range. The inset shows the contribution of material dispersion from $1 \rightarrow 2 \mu \mathrm{m}$ : note that for this example the contribution of the waveguide dispersion is negligible. We expect the waveguide component of the dispersion to become much more significant when larger holes or smaller structures are considered.

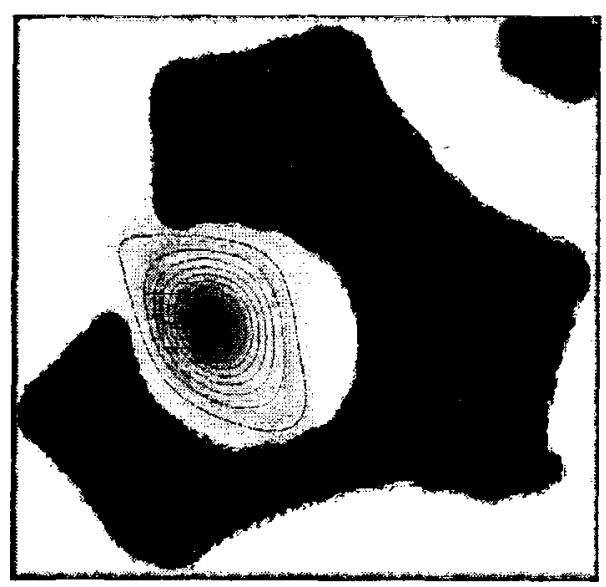

(a) Theoretical Mode Profile

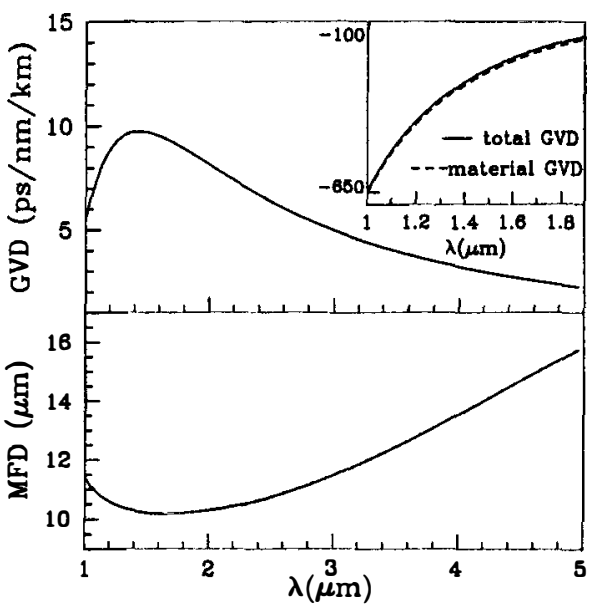

(b) Calculated Dispersion

Fig. 2. (a) The (leaky) fundamental mode guided by the fibre in Fig. 1a is superimposed on the transverse fibre profile. (b) MFD and waveguide GVD for GLS fibre with $1 \mu \mathrm{m}$ holes separated by $5 \mu \mathrm{m}$ over the GLS transmission window. The inset shows the material and net dispersion over the wavelength range for which data is available.

In conclusion, our results demonstrate the feasibility of extending microstructured fibre technology to compound glass, providing great flexibility and practical advantages for the development of fibre devices in the 0.5-5 micron wavelength range. Of particular interest will be the development of highly nonlinear optical fibres at $1.5 \mu \mathrm{m}$ which could potentially have an effective nonlinearity 10,000 times that of conventional step index fibres due to a combination of the high intrinsic nonlinearity of GLS and the small mode sizes available in microstructured fibres. In addition the large refractive index of GLS should make the fabrication of photonic bandgap fibres[7] considerablely easier in GLS than in silica.

\section{References}

1. 'Properties, Processing and Application of Glass and Rare Earth-Doped Glasses for Optical Fibres', Parts B, C and D, ed Dan Hewak, Published by IEE, Stevenage, UK.

2. Y.D. West, et al. Fibre and Integrated Optics, 19, 229 (2000).

3. M. Guittard et al. J. Solid State Chemistry, 62 191-198 (1986)

4. P.J. Bennett, T.M. Monro and D.J. Richardson, Opt. Let.. 24, 1203 (1999).

5. T.M. Monro, et al. J. Lightwave. Tech. 17, 1093 (1999).

6. T.M. Monro, et al. J. Lightwave Technol. 18, 50 (2000).

7. R.F. Cregan, et al. Science 285, 1537 (1999). 